\title{
Targeting Th17 cells in immune diseases
}

Cell Research (2014) 24:901-903. doi:10.1038/cr.2014.92; published online 15 July 2014

Last decade has seen quick developments in the understanding of a new type of $\mathrm{T}$ lymphocytes, Th17 cells. This information is benefiting the understanding and treatment of a variety of inflammatory diseases, as suggested by a recent paper in Immunity.

After activation by antigen-presenting cells (APC), naïve, antigen-specific $\mathrm{CD}^{+} \mathrm{T}$ cells differentiate into effector $\mathrm{T}$ cells. More than two decades ago, Coffman and Mosman first discovered the heterogeneity of effector $\mathrm{T}$ cells, which were then named as Th1 or Th2 cells. Th1 and Th2 cells are differentially induced and regulate immunity against intracellular and extracellular pathogens, respectively, as well as immunopathologies such as autoimmunity and allergy. The Th1/Th2 dichotomy has dominated the field of immune regulation until five years ago when IL-17-expressing T cells were proposed to be a third lineage of helper $\mathrm{T}$ cells.

Th17 cell differentiation can be induced by the combination of TGF $\beta$ and IL-6 [1]. Inhibition of TGF $\beta$ signaling in vivo was shown to substantially reduce Th17 cell generation in experimental autoimmune encephalomyelitis (EAE) model. In support of a critical role of TGF $\beta$ signaling in T cells, deficiency in TGF $\beta$ signaling or Smad2, a transcription factor downstream of TGF $\beta$ signaling also inhibited Th17 cell generation.

It has been recently debated whether TGF $\beta$ is absolutely required for Th17 cell generation. It was first shown that murine naïve $T$ cells can be induced into Th17 cells by use of IL-1, IL-6 and IL23 in the absence of exogenous TGF $\beta$. Interestingly, Th17 cells generated un- der this condition highly expressed IL22, compared with Th17 cells induced by TGF $\beta$ and IL-6. However, anti-TGF $\beta$ antibody inhibited this differentiation, suggesting that endogenous TGF $\beta$ was necessary [2]. Subsequently, the induction of Th17 cells by use of the same cytokines was also reported by another group, but it was TGF $\beta$ independent. Moreover, Th17 cells generated in this manner produced IFN $\gamma$ and expressed transcription factor T-bet as well as CXCR3, all Th1 products. And these Th17 cells were found more potent in induction of EAE than those induced in the presence of TGF $\beta$ and IL-6 [2].

A third group found that IL-23 signaling significantly upregulates the expression of TGF $\beta 3$ in T cells [2]. TGF $\beta 3$ in the presence of IL- 6 induced the "pathogenic" Th17 signatures. TGF $\beta 3$ is regarded as a weaker cytokine than TGF $\beta 1$. Perhaps at the same concentration that TGF $\beta 1$ can induce Foxp3 expression, TGF $\beta 3$ is unable. It remains to be tested whether high dose of TGF $\beta 3$ can induce regulatory phenotypes in Th17 cells. Nonetheless, this study suggests that TGF $\beta 1$ and TGF $\beta 3$ work in concert or in sequence to promote Th17 cell differentiation. This idea has not been genetically tested yet in vivo.

These latest work add a layer of complexity to the Th17 cell biology. It can be postulated that Th17 cells come in different flavors. In TGF $\beta$-rich environment, e.g., gut [2], Th17 cells may be less pathogenic or even regulatory like, whereas during extensive inflammation under the influence of proinflammatory cytokines, they may be more pathogenic, producing cytokines such as IFN $\gamma$, GM-CSF and IL-22. What intrinsic mechanisms that differentially target different sets of genes in these two types of Th17 cell differentiation are unknown. Whether these subtypes of Th17 cells are stable and can be interswitched also needs to be addressed in the future studies.

Since its discovery, Th17 cell generation and function has been linked with many environmental conditions. For example, earlier work showed an import role of Ahr, a receptor for environmental toxins, in Th17 generation [1]. On the other hand, retinoic acid (RA) and vitamin D inhibit Th17 cell differentiation [2].

Th17 cells are frequently found in the mucosal tissues. Elegant work from Littman and his colleagues first demonstrated the importance of intestinal microbiota, in particular segmented filamentous bacteria in the generation of mucosal Th17 cells in mouse [2]. Subsequently, it was reported that the presence of these Th17-inducing bacteria determines the pathogenesis of a rheumatoid arthritis disease mouse model [2]. With exponentially increasing literature on microbiota in human health and diseases, the influence of microbiota on human Th17 cell generation and function will arise in the horizon and may suggest an unconventional way of treating human diseases via modulating their micriobiome.

Sodium chloride salt has been associated with human cardiovascular diseases. Lately, high salt uptake was shown to promote autoimmunity by increasing Th17 cell generation [2]. Mechanistically, it was shown that expression of salt-sensing kinase SGK1 can be induced by IL-23 [2]. Loss of 
SGK1 resulted in protection to EAE model. This pathway may thus be targeted in human diseases.

STAT3 has been reported to be a crucial component of IL-6-mediated Th17-cell regulation [1]. Importantly, STAT3 deficiency greatly decreased the expression of ROR $\gamma t$ and ROR $\alpha$, which are now known to be Th17-cell lineagespecific transcription factors [1]. These results indicate an essential function of STAT3 in the global regulation of Th17cell gene expression programs, possibly through the induction of lineage-specific transcription factors. Consistently, in human hyper-IgE patients with STAT3 mutation, Th17 differentiation was found to be defective [3].

Th-cell lineage commitment is mediated by lineage-specific transcription factors. Similar to T-bet in Th1 cells and GATA3 in Th2 cells, two RA-related orphan receptor were recently discovered to regulate Th17-cell differentiation. ROR $\gamma \mathrm{t}$ has been identified as a candidate master regulator that drives Th17cell lineage differentiation [1]. ROR $\alpha$ is also reported to be expressed by Th17 cells; ROR $\alpha$ expression was induced by TGF $\beta$ and IL-6 in a STAT3-dependent manner [1]. Compound mutations in both factors completely inhibited Th17-cell differentiation in vitro and in vivo and entirely suppressed the development of EAE [1]. Thus, ROR $\alpha$ and ROR $\gamma t$ have similar and redundant functions.

How ROR $\gamma$ regulates gene expression is not completely understood. Ciofani et al. [4] have recently examined the genes directly bound by ROR $\gamma$. Interestingly, multiple ROR $\gamma$-binding sites were identified in the $I L-17 / I L-17 F$ gene locus. To test the function of these elements in the transcriptional regulation, Wang et al. [5] deleted one cis element, CNS2 in the $I L-17 / I L-17 F$ gene locus and found that ROR $\gamma$-dependent $I L-17$ transcription was almost completely suppressed. Moreover, epigenetic activation of the gene locus was affected, though how ROR $\gamma$ interacts with the epigenetic machineries remains unknown at this stage.

Several years ago, it was reported that Th17 cells generated in vitro can convert to other types of T cells. Nurieva et al. [6] showed that Th17 cells after one round of differentiation can readily become Treg, Th1 and Th2 cells in vitro and convert to Th1 cells in lymphopenic environments. Th17-to-Th1 conversion was simultaneously reported by two groups to be necessary for Th17 cells to induce type 1 diabetes [2].

Whether Th17 cells are plastic was analyzed by Stockinger and her colleagues using a fate-mapping strategy [2]. IL-17A-producing Th17 cells are barely detectable in unmanipulated mice, according to Stockinger and colleagues using an IL-17A reporter system [2]. They generated mice with Cre under control of IL-17A promoter. These mice were then crossed with reporter mice expressing eYFP following Cre upregulation. Any cell ever expressing IL-17A would be marked as eYFP positive. When the reporter mice were challenged with myelin oligodendrocyte peptide to induce EAE, Th17 cells were gradually induced and represented the major IL-17A-expressing cell population infiltrating in spinal cord of diseased mice. The co-existence of eYFP $^{+} \mathrm{IL}-17 \mathrm{~A}^{-} \mathrm{CD} 4^{+}$cells together with eYFP $^{+} \mathrm{IL}-17 \mathrm{~A}^{+} \mathrm{CD} 4^{+}$cells suggests the plasticity of Th17 cells under autoimmune inflammatory conditions. On the contrary, Th17 cells generated during acute inflammation against Candida albicans maintained their signature cytokine expression, with the majority of which were eYFP ${ }^{+} I L-17 \mathrm{~A}^{+}[2]$. More recently, the same group [7] also reported that Th17 cells can convert to $\mathrm{Bcl}^{+}$follicular helper $\mathrm{T}(\mathrm{Tfh})$ cells in intestinal Peyer's patches, although there was hardly any association between Tfh and Th17 cells in the peripheral lymphoid organs.

From above, Th17 cells can maintain their phenotypes under some circumstances but are plastic in others.
The extrinsic and intrinsic factors that determine Th17 cell maintenance and reprogramming will need to be sought out, which may have implications in immune diseases.

Since its discovery, Th17 cells have been linked with autoimmune diseases; inhibition of Th17 cell generation or function is protective in EAE. Lately, clinical trials with anti-IL-17 antibody have proven targeting this pathway to be a novel treatment of human inflammatory diseases, including rheumatoid arthritis, uveitis and psoriasis [8].

Additional diseases may use the same strategy. For example, Th17 cells have been functionally linked with asthma and COPD $[9,10]$. In addition, some cancers, especially colon cancer, have been shown to depend on Th17 cells or IL-17 [11].

How to target Th17 cells is certainly a good technical question. One may consider inhibiting IL-17 function, especially in diseases that depend on this cytokine, with the advantage of neutralizing it derived from cells more than Th17 cells, $\gamma \delta \mathrm{T}$ cells and innate lymphocytes, etc. Or to inhibit multiple effector molecules derived from Th17 cells, ROR $\gamma$ is a desirable target, as multiple inhibitors of ROR $\gamma$ have been shown to be effective in EAE, including a recent study published in Immunity [12]. Development of multiple strategies may give physicians more and wiser choices in treatment of patients.

Th17 cell is 8 years old! There has been very rapid understanding on the regulation of Th17 cell differentiation and a number of factors have been identified to be important in this process. How these factors work individually and together still require further elucidation. Another important emerging issue is the plasticity of T cells. Although redifferentiation of Th17 cells has been observed in several circumstances, the physiological relevance and significance are still unclear. Nonetheless, our current understanding on Th17 cells and future development in the field will 
assist more effective and rational treatment of human diseases, as supported by the encouraging results from clinical trials of multiple Th17-inhibiting agents.

\section{Chen Dong ${ }^{1}$}

${ }^{I}$ Tsinghua University School of Medicine, Beijing 100084, China

Correspondence: Chen Dong

E-mail: cdonglab@hotmail.com

\section{References}

1 Dong C. Nat Rev Immunol 2008; 8:337-348.

2 Peters A, Lee Y, Kuchroo VK. Curr Opin Immunol 2011; 23:702-706.

3 Milner JD, Brenchley JM, Laurence A, et al. Nature 2008; 452:773-776.

4 Ciofani M, Madar A, Galan C, et al. Cell 2012; 151:289-303.

5 Wang X, Zhang Y, Yang XO, et al. Immunity 2012; 36:23-31.

6 Nurieva R, Yang XO, Chung Y, et al. J Immunol 2009; 182:2565-2568.
7 Hirota K, Turner JE, Villa M, et al. Nat Immunol 2013; 14:372-379.

8 Hueber W, Patel DD, Dryja T, et al. Sci Transl Med 2010; 2:52ra72.

9 Yang XXO, Chang SH, Park H, et al.J Exp Med 2008; 205:1063-1075.

10 Shan M, Yuan X, Song LZ, et al. Sci Transl Med 2012; 4:117ra119.

11 Tong Z, Yang XO, Yan $\mathrm{H}$, et al. PLoS One 2012; 7:e34959.

12 Xiao S, Yosef N, Yang J, et al. Immunity 2014; 40:477-489. 